\title{
Improving Birth Weight Measurement and Recording Practices in Kenya and Tanzania: A Prospective Intervention Study with Historical Controls
}

\author{
Alloys KOloo \\ Kenya Medical Research Institute \\ Evance Godfrey \\ Muhimbili National Hospital \\ Annariina M Koivu \\ Tampere University: Tampereen Yliopisto \\ Hellen C. Barsosio \\ KEMRI: Kenya Medical Research Institute \\ Karim Manji \\ MUHAS: Muhimbili University of Health and Allied Sciences \\ Veneranda Ndesangia \\ MUHAS: Muhimbili University of Health and Allied Sciences \\ Fredrick Omiti \\ KEMRI: Kenya Medical Research Institute \\ Mohamed Bakari Khery \\ MUHAS: Muhimbili University of Health and Allied Sciences

\section{Everlyne D. Ondieki} \\ KEMRI: Kenya Medical Research Institute

\section{Simon Kariuki} \\ KEMRI: Kenya Medical Research Institute

\section{Feiko 0. ter Kuile} \\ Liverpool School of Tropical Medicine

\section{R Matthew Chico} \\ London School of Hygiene \& Tropical Medicine \\ Nigel Klein \\ Institute of Child Health
}

Otto Heimonen

Tampere University: Tampereen Yliopisto

\section{Per Ashorn}

Tampere University: Tampereen Yliopisto 


\section{Ulla Ashorn}

Tampere University: Tampereen Yliopisto

Pieta Näsänen-Gilmore ( $\nabla$ pieta.nasanen-gilmore@tuni.fi )

Tampere University: Tampereen Yliopisto https://orcid.org/0000-0002-6886-4126

\section{Research}

Keywords: Low birth weight (LBW), measurement, accuracy, digital scales, data quality, low- and middleincome countries (LMIC)

Posted Date: March 8th, 2022

DOI: https://doi.org/10.21203/rs.3.rs-658362/v2

License: (c) (i) This work is licensed under a Creative Commons Attribution 4.0 International License.

Read Full License 


\section{Abstract \\ Background}

Low birth weight (LBW) is a significant public health concern given its association with early-life mortality and other adverse health consequences that can impact the entire life-cycle. In many countries, accurate estimates of LBW prevalence are lacking due to inaccuracies in collection and gaps in available data. Our study aimed to determine LBW prevalence among facility-born infants in selected areas of Kenya and Tanzania, and to assess whether the introduction of an intervention to improve the accuracy of birthweight measurement would result in a meaningfully different estimate of LBW prevalence than current practice.

\section{Methods}

We carried out a historically-controlled intervention study in 22 health facilities in Kenya and three health facilities in Tanzania. The intervention included: provision of high-quality digital scales, training of nursing staff on accurate birth weight measurement, recording and scale calibration practices, and quality maintenance support that consisted of enhanced supervision and feedback (prospective arm). The historically-controlled data were birth weights from the same facilities recorded in maternity registers for the same calendar months from the previous year measured using routine practices and manual scales. We calculated mean birth weight ( $95 \%$ confidence interval $\mathrm{Cl}$ ), mean difference in LBW prevalences and respective risk ratio $(95 \% \mathrm{Cl})$ between study arms.

\section{Results}

Between October 2019 and February 2020, we prospectively collected birth weights from 8,441 newborns in Kenya and 4,294 in Tanzania. Historical data were available from 9,318 newborns in Kenya and 12,007 in Tanzania. In the prospective sample, the prevalence of LBW was $12.6 \%$ (95\% confidence intervals [CI]: $10.9 \%-14.4 \%)$ in Kenya and $18.2 \%(12.2 \%-24.2 \%)$ in Tanzania. In the historical sample, the corresponding prevalence estimates were $7.8 \%(6.5 \%-9.2 \%)$ and $10.0 \%$ (8.6\%-11.4\%). Compared to the retrospective sample, the LBW prevalence in the prospective sample was 4.8\%-points (3.2\%-6.4\%) higher in Kenya and 8.2\%-points (2.3\%-14.0\%) higher in Tanzania, corresponding to a risk ratio of 1.61 (1.38-1.88) in Kenya and 1.81 (1.30-2.52) in Tanzania.

\section{Conclusion}

Routine birth weight records under-estimate the risk of LBW among facility born infants in Kenya and Tanzania. The quality of birth-weight data can be improved by a simple intervention consisting of provision of digital scales and supportive training. 


\section{Background}

Low birth weight (LBW), defined by the World Health Organization (WHO) as a birth weight less than 2,500 grams (1), is a major contributor to neonatal mortality (2-4). The LBW infants who survive infancy are at increased risk of long-term sequelae, including neurodevelopmental problems (5); stunting (6); respiratory disorders (7); lower IQ (8); and adult-onset chronic diseases (9-12). Additionally, when LBW girls become mothers, they are more likely to deliver LBW infants themselves (13). Thus, reducing the incidence of LBW has public health implications that are lifelong and inter-generational.

Globally, approximately $15 \%$ of live births are LBW, with the highest burden in southern Asia and subSaharan Africa (14). However, the true magnitude is likely underestimated due to poor data coverage and quality. Poor data coverage may result from home births where newborn weight is rarely measured or recorded (15-17) or when the neonate is frail, ill, or dies (15). Alternatively, newborns may be weighed, but the measurement is not recorded in routine data sources such as health cards (18). Birth weight data may also be inaccurately recorded for various reasons. A common preference for the terminal digit 0 or 5 has been described $(19,20)$ and heaping of birth weight data, which involves rounding birth weights to the closest 100- or 500-gram interval. For example, infants weighing 2,490 grams are recorded as 2,500 grams. This practice leads to inaccurate estimates of $\operatorname{LBW}(21,22)$ at the individual and population levels $(15,20,23)$. Other barriers to accurate birth weight data are random and systematic measurement errors (24), including delays in birth-weight measurement until several days after birth $(24,25)$, or subtracting the estimated weight of clothes after measuring a dressed or swaddled newborn (21); inaccurate, unavailable or inaccessible scales $(21,24,26)$; lack of standardized technical weighing protocols $(21)$; or poor calibration of scales (14). Complicated register design may further contribute to inaccuracies in birthweight data (27). Finally, various health system or sociocultural factors, including limited understanding of why the data are collected, may underpin sub-optimal data quality and use (28).

Accurate birth weight measurement serves as a guide to appropriate care for newborns. It is also a pivotal metric in monitoring neonatal outcomes at the population level and tracking national, regional, and global progress towards the Every Newborn Action Plan (29), the Global Nutrition Plan (1) and Sustainable Development Goals (30). These global initiatives may be particularly important for LMICs, the same countries that face the most barriers to reliable LBW data. Against this background, we estimated the prevalence of LBW among infants born at selected time periods in two LMICs and evaluated a simple intervention designed to improve LBW measurements with the aim of answering two research questions:

1. What is the prevalence of LBW among infants born in health facilities in selected health areas in Kenya and Tanzania?

2. Will the introduction of a support package (use of improved scales, training and enhanced staff supervision and feedback) result in different LBW prevalence estimate compared to current practices in the same health facilities? 
By addressing these questions, we sought to contribute to the improved understanding of existing newborn weighing methods in low-resource settings and improve the accuracy of measurements with simple intervention.

\section{Methods}

\subsection{Study design}

We conducted a prospective study with historical controls at 22 health facilities in Kenya and three health facilities in Tanzania to improve birth weight measurement and recording practices (Figure 1). The prospective component was conducted for 4.5 months from October 2019 to February 2020, targeting all neonates born during the study period (prospective arm). Historical birthweight data consisted of previously recorded birth weight data for all births from the same selected health facilities during the same calendar months of the preceding year (historical arm).

\subsection{Study context}

Kenya and Tanzania are representative of LMICs where access to accurate and timely LBW data is a challenge $(17,21,31)$. In Kenya, the latest country-level LBW estimate, $11.5 \%$ (95\% Cl: $8.9-14.5)$ of live births, is from 2015 (18). The Kenya Demographic and Health Survey 2014 estimated LBW prevalence to be $7.6 \%$ among newborns with a reported birth weight (32). In Tanzania, the country prevalence of LBW was estimated at $10.5 \%$ (95\% Cl: 8.1-13.4) in 2015 (18). Additionally, Tanzanian record-based studies have previously reported prevalence rates of 9.5-20.5\% between 2000-2010 and 2010-2015, respectively, depending on the study setting and population $(33,34)$, suggesting considerable in-country variation.

\subsection{Study sites}

The study sites were selected to represent a range of delivery facilities in urban and rural settings. These sites varied in numbers of births per year, infrastructure, average county-level birth rate, and staffing and supervision. We selected study facilities if their maternity registers contained complete birth information, including birth date, birth weight and sex of the newborns for the year preceding the study period. In Kenya, the study was conducted in 22 rural health facilities in four counties (Homa Bay, Siaya, Migori, and Kisumu). In Tanzania, the study was conducted in two referral hospitals (Amana and Temeke) and one health centre (Mbagala Rangitatu) in suburban Dar es Salaam (Table 1). 
Table 1

Data collection sites in study countries

\begin{tabular}{|c|c|c|c|c|}
\hline Country & County / site & $\begin{array}{l}\text { No. of health } \\
\text { facilities included }\end{array}$ & $\begin{array}{l}\text { Type of health } \\
\text { facilities by level }\end{array}$ & $\begin{array}{l}\text { No. of deliveries } \\
\text { per month }\end{array}$ \\
\hline \multirow[t]{4}{*}{ Kenya } & Kisumu County & 8 & $3,4,5$ & 680 \\
\hline & Siaya County & 5 & $3,4,5$ & 410 \\
\hline & Homa Bay County & 5 & $3,4,5$ & 420 \\
\hline & Migori County & 4 & $3,4,5$ & 340 \\
\hline \multirow[t]{3}{*}{ Tanzania } & $\begin{array}{l}\text { Temeke Regional } \\
\text { Referral Hospital }\end{array}$ & 1 & 4 & 1,000 \\
\hline & Mbagala Rangi & 1 & 3 & 1,000 \\
\hline & $\begin{array}{l}\text { Amana Regional } \\
\text { Referral Hospital }\end{array}$ & 1 & 4 & 900 \\
\hline \multicolumn{5}{|c|}{${ }^{1}$ The six levels of health care service delivery are 1 community, 2 dispensaries, 3 health centre, } \\
\hline
\end{tabular}

\subsection{Intervention}

The support package provided to the prospective group consisting of:

1. Provision of digital weighing scales with 10 -gram reading increment.

2. Training of health workers in scale calibration and use and in precise birth-weight recording.

3. Quality maintenance support via setting up a weekly supervision and feedback routine for senior nurses and facility managers on the intervention uptake and data quality.

4. Scheduled midpoint retraining to reinforce the effectiveness of the intervention.

The intervention was fitted into the existing newborn weighing and care routines of the selected health facilities as much as possible.

\section{Provision of scales}

Before the intervention, the facilities used traditional analogue weighing scales or hand-held scales. We provided each study facility a battery-operated digital scale (Seca 354, Seca, Hamburg, Germany) that measures in graduations of 10 grams. Larger facilities with separate operating theatres were given two scales. We also provided standard weights $(0.5 \mathrm{~kg}, 1.0 \mathrm{~kg}, 2 \mathrm{~kg}, 3 \mathrm{~kg})$ for calibration. In some facilities, official calibration stones of similar weight were already available. If these existing calibration stones were used, we ensured that they produced correct readings and were in good condition with no visible chippings, affecting the weight. 


\section{Training}

Prior to data collection, we carried out a three-day training among staff members from labor-, post-natal-, and natal wards and obstetric theatres of the selected health facilities. For the larger facilities in Kenya, we trained health facility supervisors who then trained nurses, whereas in smaller facilities in Kenya and all facilities in Tanzania, we trained nurses. Participants were provided with a detailed outline of the study, highlighting the importance of accurate birth weight measurement. Nurses were trained to weigh naked newborns within one hour of birth, using the digital scale provided on a level hard surface and recording the result with 10-gram precision. A standard operating procedure was provided to ensure uniformity of performance. We also trained the nurses to calibrate the digital scales and provided a daily calibration sheet in which the nurses were asked to record the status of calibration at the start of each working day. Scale readings within the limit of +/-20 grams were considered acceptable. In addition, nurses were asked to record all birth data into the maternity register following the existing routine practices. We tested whether the amount of training was adequate at each health facility for a period of two days following the training. We randomly selected nurses from each facility who practised daily calibration of the scales, invited mothers for routine newborn weighing and practised the improved birth weight measuring techniques using the digital scales.

\section{Quality maintenance}

Health facility supervisors and district nurses were asked to reinforce the training of health workers and midwives through enhanced supervision and feedback on-site. They were also instructed to report to the research team on the quality of work using a daily calibration log and weekly supervision logs.

Furthermore, they were asked to ensure with regularity the accuracy and completeness of the data. In Kenya, depending on the site, health facility supervisors or district nurses visited the sites once a week supported and supervised by the central research team. In Tanzania, quality maintenance was conducted by a member of the research team who visited the sites weekly.

\section{Retraining}

At the midpoint of the data collection, the central research team in each country visited the study locations to reinforce the effect of the support package by running a second training session at all sites. The training consisted of the same topics as the first session but was reduced to half a day. At smaller sites, the research team trained the nurses and midwives, whereas, at the larger sites, the research team trained the district nurses, i.e. the trainers of the nurses and midwives.

\subsection{Data collection and entry}

We collected data from maternity records for the prospective and historically-controlled groups. We obtained data on maternity ID, birth weight in grams, sex of the newborn (male/female/not recorded) and date of birth (dd, mm, yyyy). In Kenya, for prospective data, field supervisors collected data weekly and entered data into a tablet with CommCare data collection software (Dimagilnc, USA). For historical data, the field supervisors photographed the historically-controlled data and entered them into the CommCare 
application. In Tanzania, both historical and prospective data were photographed and entered into an Excel spreadsheet (Microsoft Excel, USA). In both countries, collected variables were the same, and the data were entered in the dataset by two people independently. The two datasets were linked using maternity ID and cross-checked by data managers in the research team. If any discrepancies were identified, the data were checked from the original health records.

\subsection{Statistical analyses}

We calculated mean birth weight and $95 \%$ confidence interval $(\mathrm{Cl})$. We also compared the mean birth weight between the prospective intervention group and the historical control groups using the Student's independent $t$-test (Stata 16, Stata Corp, USA). The outcome variable (birth weight) was grouped into LBW $(<2500 \mathrm{~g})$ and normal birth weight $(\geq 2500 \mathrm{~g})$ in the categorical analysis. We calculated the absolute difference and risk ratio (and 95\% Cls) in LBW prevalence between the prospective and historical arms. The analyses were adjusted for the cluster (health facility). Additionally, due to the known practice of digit preference resulting in heaping of birth weight data on multiples of $500 \mathrm{~g}$, we calculated an adjusted LBW prevalence by reallocating $25 \%$ of infants with an exact birth weight of $2500 \mathrm{~g}$ to the LBW category. This adjustment method has been used in previous studies $(23,27)$.

\section{Results}

Between October 2019 and February 2020, we prospectively collected birth weights from 8,441 newborns in Kenya and 4,294 in Tanzania. Historical data were available from 9,318 newborns in Kenya and 12,007 in Tanzania (Table 2). The prospectively recorded birth weights ranged from 600 to $5,890 \mathrm{~g}$ in Kenya and 700 to 4,600g in Tanzania. The birth weights based on historical data ranged from 650 to $5,700 \mathrm{~g}$ in Kenya and 690 to 5,500g in Tanzania (Figure 2).

The mean (SD) birth weight in Kenya was $3080 \mathrm{~g}$ (590) in the prospective sample and $3190 \mathrm{~g}(570)$ in the historical sample. In Tanzania, the respective values were 2,990g (590) and 3,030g (530). Compared to the historical sample, the mean birth weight in the prospective sample was $110 \mathrm{~g}$ (95\% Cl: 100-130) lower in Kenya and 40g (95\% Cl: 20-60) lower in Tanzania.

In Kenya, the prevalence of LBW assessed prospectively ( $\mathrm{N}=8,441)$ was $12.6 \%$ (95\% Cl: $10.9 \%-14.4 \%)$ compared to $7.8 \%(6.5 \%-9.2 \%)$ in the historical data $(\mathrm{N}=9,318)$ (absolute difference $4.8 \%$-point, $(95 \% \mathrm{Cl}$ : $3.2 \%-6.4 \%)$, risk ratio $1.61(1.38-1.88)$ ). The corresponding figures in Tanzania were $18.2 \%(95 \% \mathrm{Cl}$ : $12.2 \%-24.2 \%)$ prospectively $(\mathrm{N}=4,294)$ and $10.0 \%(95 \% \mathrm{Cl}: 8.6 \%-11.4 \%)$ retrospectively $(\mathrm{N}=12,007)$ (absolute difference $8.2 \%$-point ( $95 \% \mathrm{Cl}: 2.3 \%-14.0 \%)$, risk ratio $1.81(1.30-2.52)$ ). A sensitivity analysis using adjusted birth weights by reallocating $25 \%$ of infants with an exact birth weight of $2,500 \mathrm{~g}$ to the LBW category gave essentially similar results in both countries (Table 2).

Table 2. Prevalence of birth weights below 2,500g (LBW) in Kenya and Tanzania in prospective and historical sample. 


\begin{tabular}{|c|c|c|c|c|}
\hline & $\begin{array}{l}\text { Prospective } \\
\text { Sample } \\
(95 \% \mathrm{CI})\end{array}$ & $\begin{array}{l}\text { Historical } \\
\text { Sample } \\
(95 \% \mathrm{CI})\end{array}$ & $\begin{array}{l}\text { Absolute } \\
\text { difference in } \\
\text { LBW } \\
\text { prevalence } \\
(95 \% \mathrm{CI})\end{array}$ & $\begin{array}{l}\text { Risk ratio } \\
(95 \% \mathrm{CI})\end{array}$ \\
\hline \multicolumn{5}{|l|}{ Kenya } \\
\hline $\begin{array}{l}\text { Number of } \\
\text { births }\end{array}$ & 8,441 & 9,318 & N/A & $\mathrm{N} / \mathrm{A}$ \\
\hline $\begin{array}{l}\text { Proportion of birth } \\
\text { weights }<2500 \mathrm{~g}\end{array}$ & $\begin{array}{l}12.6 \% \\
(10.9 ; 14.4)\end{array}$ & $\begin{array}{l}7.8 \% \\
(6.5 ; 9.2)\end{array}$ & $\begin{array}{l}4.8 \% \\
(3.2 ; 6.4)\end{array}$ & $\begin{array}{l}1.61 \\
(1.38 ; 1.88)\end{array}$ \\
\hline $\begin{array}{l}\text { Adjusted LBW\% } \\
\text { proportion of birth } \\
\text { weights }<2500 \mathrm{~g}^{*}\end{array}$ & $\begin{array}{l}12.9 \% \\
(11.2 ; 14.6)\end{array}$ & $\begin{array}{l}8.5 \% \\
(7.1 ; 9.9)\end{array}$ & $\begin{array}{l}4.3 \% \\
(2.7 ; 6.0)\end{array}$ & $\begin{array}{l}1.51 \\
(1.29 ; 1.77)\end{array}$ \\
\hline \multicolumn{5}{|l|}{ Tanzania } \\
\hline $\begin{array}{l}\text { Number of } \\
\text { observations }\end{array}$ & 4,294 & 12,007 & N/A & N/A \\
\hline $\begin{array}{l}\text { Proportion of birth } \\
\text { weights }<2500 \mathrm{~g}\end{array}$ & $\begin{array}{l}18.2 \% \\
(12.2 ; 24.2)\end{array}$ & $\begin{array}{l}10.0 \% \\
(8.6 ; 11.4)\end{array}$ & $\begin{array}{l}8.2 \% \\
(2.3 ; 14.0)\end{array}$ & $\begin{array}{l}1.81 \\
(1.30 ; 2.52)\end{array}$ \\
\hline $\begin{array}{l}\text { Adjusted LBW\% } \\
\text { proportion of birth } \\
\text { weights }<2500 \text { g* }\end{array}$ & $\begin{array}{l}18.5 \% \\
(12.7 ; 24.2)\end{array}$ & $\begin{array}{l}11.4 \% \\
(9.9 ; 12.9)\end{array}$ & $\begin{array}{l}7.1 \\
(1.9 ; 12.3)\end{array}$ & $\begin{array}{l}1.62 \\
(1.23 ; 2.13)\end{array}$ \\
\hline
\end{tabular}

*Adjusted LBW prevalence was calculated after re-allocating $25 \%$ of $2500 \mathrm{~g}$ infants to be LBW

\section{Discussion}

The purpose of this study was to determine a prevalence of LBW among infants born in selected health facilities in two LMIC countries and to assess if a simple intervention to improve the accuracy of birth weight measurement would produce a different estimate of LBW prevalence than the current practice. The provision of new digital scales, training of nurses, and quality maintenance support resulted in a higher estimate of LBW prevalence: 4.8 percentage points (from 7.8-12.6\%) in Kenya and 8.2 percentage points (from 10.0-18.2\%) in Tanzania compared to historical sample produced using routine practices in a sample of approximately 34,000 facility births.

The validity of the findings could theoretically have been affected by variation in birth weight measurement in the multiple study facilities, but we minimized this problem by enhanced supervision, communication and clear accountability structures. Moreover, the study facilities might be considered "high performers" given that they contained complete birth information from the previous year. The differences between prospective and historical data might have been greater in facilities with incomplete 
birth information. In Tanzania, the number of births was lower in the prospective sample than the historical one because a new birthing facility was opened in the study area during the period of prospective data collection. This might theoretically have led to the referral of low-risk mothers to this new facility and an increase in the proportion of high-risk deliveries in the study facilities, and thus potentially have contributed to an increase in the proportion of LBW infants in our prospective sample. There was, however, no public recommendation to concentrate or refer certain types of deliveries to specific facilities. Because of this, and the consistency of the findings, we believe that our findings are valid and indicate that routine birth weight recording has produced considerable under-estimates in the prevalence of LBW.

Observer errors have been reported in birth-weight research (36). Weights ending in multiples of 100 or 500 grams tend to be preferred, resulting in a heaping of birth weight measurements $(15,19,20)$. This affects LBW estimates, particularly in LMICs (22). While heaping is less common in birth-weight data from health cards than from maternal recall, the practice can still significantly affect LBW estimates. For example, in a comparison of six LMICs, $10-64 \%$ of birth weights on health cards were recorded in multiples of $500 \mathrm{~g}$ or $1 / 2 \mathrm{lb}(22)$. To address this, we frequently highlighted in training the importance of precision and introduced digital scales, which reduces measurement heaping $(27,37)$. While there was still some heaping, even in our prospective sample, it did not significantly affect the estimate of the LBW prevalence or the impact of the study intervention on it, as evidenced by the sensitivity analysis.

The 12.4\% LBW prevalence in Kenya and 18.2\% in Tanzania are higher than the UNICEF reported national prevalence rates from 2015 (11.5\% for Kenya and 10.5\% for Tanzania (18)) and higher than the estimates in Demographic Health Surveys for the study locations $(32,38)$, but roughly in line with the regional rate for sub-Saharan Africa which was estimated to be 12.2-17.2\% in 2015 (14). Demographic Health Surveys are likely to underestimate the prevalence of LBW due in part to accuracy issues previously discussed, reliance in some cases on maternal recall and the notable proportion of newborns who are not weighed (15).

We did not identify other controlled studies to achieve more precise LBW estimates through an intervention targeted at the facility level. An Indian hospital-based study weighed 859 live births using analog and digital scales and found that significantly more newborns weighed exactly $2,500 \mathrm{~g}$ on analog versus digital scales. The prevalence of LBW by digital scale $(29.5 \%)$ was significantly higher compared to the analogue device (23.0\%) (37). Data quality interventions using various forms of support, including training, reviews, audits and feedback, have improved accuracy, completeness, timeliness, and other quality aspects in maternal and newborn health data in LMICs (39-41). However, these types of studies do not typically provide before and after LBW estimates. The EN-BIRTH study examined labor and delivery ward register data availability, quality, and utility and identified significant heaping of birth weights in Bangladesh, Nepal, and Tanzania (42). However, the study design included no intervention to address the deficiencies in LBW data quality. The study also compared the birth-weight data in hospital registers and women's report at exit interview survey (27) and found that the register-based LBW rate was $14.9 \%$ for the three countries, and the rate was slightly higher and more specific and sensitive than 
survey-based rate. The qualitative component of the EN-BIRTH study explored barriers and enablers to weighing birth in Temeke Hospital, which is one of the sites of the current study. Our intervention directly addressed many of the gaps they reported, including the lack of precise equipment and standardized technical weighing protocols. A recent study from Ethiopia reports the effect of data quality intervention on LBW prevalence before and after the intervention and provides interesting qualitative insights into the success of the intervention (43).

The LBW prevalences reported in the current study are not generalizable beyond the study areas and cannot be taken as indicators of national LBW prevalence in Kenya and Tanzania. This is because data collection took place only at health facilities and included no data from home deliveries that remain common in both countries. Furthermore, the study samples were not designed to be nationally representative, as they represented only limited numbers of geographic areas and only some types of maternity wards. However, whilst these issues obviously limit the generalizability of the reported LBW prevalences, they do not affect the main conclusions of the study.

\section{Conclusions}

There has been a call for research to establish the efficacy and feasibility of interventions to improve the quality of birth-weight data (21). Our study demonstrated that routine birth weight data markedly underestimates LBW prevalence. Furthermore, it showed that a simple intervention introducing only modest changes in existing daily practices at health facilities can lead to significantly more accurate birth-weight data in low resource settings and thus contribute to more precise LBW estimates. This relatively low-cost intervention, which does not require excessive training, is readily deliverable in LMICs beyond the study countries and has the potential to improve birth weighing and data recording practices globally.

\section{Abbreviations}

BW - Birth weight

$\mathrm{Cl}$ - Confidence interval

IRB - Institution Review Board

KEMRI - Kenya Medical Research Institute

LBW - Low birth weight

LMIC - Low- and middle income country

MUHAS - Muhimbili University of Health and Allied Sciences

MNH - Muhimbili National Hospital 
$\mathrm{MoH}$ - Ministry of Health

NIMR - National Institute of Medical Research

WHO - World Health Organization

\section{Declarations}

\section{Ethics approval and consent to participate}

The study was approved the Ethical Committee of Muhimbili University of Health and Allied Sciences (MUHAS) Institution Review Board (IRB) and National Institute of research (NIMR). In Kenya approval was obtained from Jaramogi Oginga Odinga Teaching and Referral Hospital Ethics and Review Committee (JOOTRH-IERC). The methods of this study followed the guidelines and the regulations of the Declaration of Helsinki. No individual consent were sought, since investigators only studied data obtained from hospital registries and no data on personal identifiers was collected. This practise was approved by institutional review boards at both countries: in Tanzania by Muhimbili University of Health and Allied Sciences (MUHAS) Institution Review Board (IRB) and the National Institute of research (NIMR), and in Kenya by Jaramogi Oginga Odinga Teaching and Referral Hospital Ethics and Review Committee (JOOTRH-IERC).

\section{Consent for publication}

Not applicable

\section{Availability of data and materials}

The datasets used and/or analyzed during the current study are available from the corresponding author on reasonable request

\section{Competing interests}

The authors declare that they have no competing interests.

\section{Funding}

The study was funded by the Children's Investment Fund Foundation (CIFF)

\section{Authors' contributions}

AK and EK are joint-first authors and made substantial contributions to the data acquisition and data analysis and drafting of the manuscript. Additionally, AK contributed to the creation of new software applied in the data acquisition.

AMK substantively contributed to the interpretation of data and drafting the manuscript. 
SK, HCB, KM, FT, RMC and NK made significant contributions to the design of the study.

VN, FO, MBK, and EDO were responsible for organizing the study intervention and data collection.

OT significantly contributed to the data analysis.

PA and UA substantially contributed to the conception and design of the study, data analysis, interpretation of data, and manuscript revision.

PNG supervised the project and significantly contributed to the conception, design of the study, data acquisition and data analysis, and interpretation of data. Together with AK and EK, she wrote the first draft of the manuscript.

All authors critically appraised the manuscript and approved the final version of it.

\section{Acknowledgments}

We wish to acknowledge the staff of the maternity ward and management team at all the facilities we worked in for their support and hard work during the study and the funder Children's Investment Fund Foundation (CIFF). We also thank Sydney Shanghai for efficient data input in Tanzania.

\section{Author Details}

${ }^{1}$ Kenya Medical Research Institute/Centre for Global Health Research, Kisumu, Kenya

${ }^{2}$ Muhimbili National Hospital, Dar es Salaam, Tanzania (MNH)

${ }^{3}$ Muhimbili University of Health and Allied Sciences in Dar es Salaam, Tanzania (MUHAS)

${ }^{4}$ Faculty of Medicine and Health Technology, Tampere University, Tampere, Finland

${ }^{5}$ Liverpool School of Tropical Medicine, Liverpool, UK

${ }^{6}$ Department of Disease Control, Faculty of Infectious \& Tropical Diseases, London School for Hygiene and Tropical Medicine, London, UK

${ }^{7}$ University College London, Great Ormond Street, Institute of Child Health, London, UK

${ }^{8}$ Department of Paediatrics, Tampere University Hospital, Finland

${ }^{\mathbb{F}}$ Finnish National Institute for Health and Welfare, Department for Public Health and Welfare, Public Health Unit, Helsinki, Finland. 
${ }^{1}$ Kenya Medical Research Institute, Centre for Global Health Research, Kisumu, Kenya

${ }^{2}$ Muhimbili National Hospital, Dar es Salaam, Tanzania

${ }^{3}$ Muhimbili University of Health and Allied Sciences, Dar es Salaam, Tanzania

${ }^{4}$ Tampere University, Faculty of Medicine and Health Technology, Tampere, Finland

${ }^{5}$ Liverpool School of Tropical Medicine, Liverpool, L3 5QA, UK

${ }^{6}$ London School of Hygiene \& Tropical Medicine, Department of Disease Control, Faculty of Infectious \& Tropical Diseases, London, UK

${ }^{7}$ UCL Great Ormond Street Institute of Child Health

${ }^{8}$ Tampere University Hospital, Department of Paediatrics, Tampere, Finland

${ }^{\mathbb{Q}}$ Finnish Institute for Health and Welfare, Helsinki, Finland

\section{References}

1. World Health Organization. Global nutrition targets 2025: low birth weight policy brief (WHO/NMH/NHD/14.5). 2014.

2. Lawn JE, Blencowe H, Oza S, You D, Lee AC, Waiswa P, et al. Every Newborn: progress, priorities, and potential beyond survival. Lancet 2014;384(9938):189-205.

3. Eshete A, Alemu A, Zerfu TA. Magnitude and Risk of Dying among Low Birth Weight Neonates in Rural Ethiopia: A Community-Based Cross-Sectional Study. Int. J. Pediatr. 2019.

4. McCormick MC. The contribution of low birth weight to infant mortality and childhood morbidity. N Engl J Med 1985;312(2):82-90.

5. Baschat AA. Neurodevelopment after fetal growth restriction. Fetal Diagn Ther 2014;36(2):136-142.

6. Christian P, Lee SE, Donahue Angel M, Adair LS, Arifeen SE, Ashorn P, et al. Risk of childhood undernutrition related to small-for-gestational age and preterm birth in low-and middle-income countries. Int J Epidemiol 2013;42(5):1340-1355.

7. Mebrahtu TF, Feltbower RG, Greenwood DC, Parslow RC. Birth weight and childhood wheezing disorders: a systematic review and meta-analysis. J Epidemiol Community Health 2015;69(5):500508.

8. Gu H, Wang L, Liu L, Luo X, Wang J, Hou F, et al. A gradient relationship between low birth weight and IQ: A meta-analysis. Scientific reports (Nature Publisher Group) 2017;7(1):1-13.

9. Belbasis L, Savvidou MD, Kanu C, Evangelou E, Tzoulaki I. Birth weight in relation to health and disease in later life: an umbrella review of systematic reviews and meta-analyses. BMC Med 2016 -09-28;14(1). 
10. Alexander BT, Dasinger JH, Intapad S. Effect of low birth weight on women's health. Clin Ther 2014;36(12):1913-1923.

11. Osmond C, Barker DJ. Fetal, infant, and childhood growth are predictors of coronary heart disease, diabetes, and hypertension in adult men and women. Environ Health Perspect 2000;108(suppl 3):545-553.

12. Godfrey KM, Barker DJ. Fetal nutrition and adult disease. Am J Clin Nutr 2000;71(5):1344S-1352S.

13. United Nations Children's Fund and World Health Organization. Low Birthweight: Country, regional and global estimates. UNICEF 2004.

14. Blencowe H, Krasevec J, de Onis M, Black RE, An X, Stevens GA, et al. National, regional, and worldwide estimates of low birthweight in 2015, with trends from 2000: a systematic analysis. Lancet Glob Health 2019;7(7):e849-e860.

15. Biks GA, Blencowe H, Hardy VP, Geremew BM, Angaw DA, Wagnew A, et al. Birthweight data completeness and quality in population-based surveys: EN-INDEPTH study. Popul Health Metr 2021;19(1):17.

16. Rahman MS, Howlader T, Masud MS, Rahman ML. Association of Low-Birth Weight with Malnutrition in Children under Five Years in Bangladesh: Do Mother's Education, Socio-Economic Status, and Birth Interval Matter? PLoS One 2016;11(6):1-16.

17. Gisore P, Shipala E, Otieno K, Rono B, Marete I, Tenge C, et al. Community based weighing of newborns and use of mobile phones by village elders in rural settings in Kenya: a decentralised approach to health care provision. BMC Pregnancy Childbirth 2012;12(1):1-8.

18. United Nations Children's Fund (UNICEF), World Health Organization (WHO). UNICEF-WHO Low birthweight estimates: Levels and trends 2000-2015. Geneva: World Health Organization; 2019 Licence: CC BY-NC-SA 3.0 IGO.

19. Edouard L, Senthilselvan A. Observer error and birthweight: digit preference in recording. Public Health 1997;111(2):77-79.

20. Nigatu D, Haile D, Gebremichael B, Tiruneh YM. Predictive accuracy of perceived baby birth size for birth weight: a cross-sectional study from the 2016 Ethiopian Demographic and Health Survey. BMJ Open 2019;9(12).

21. Gladstone ME, Salim N, Ogillo K, Shamba D, Gore-Langton GR, Day LT, et al. Birthweight measurement processes and perceived value: qualitative research in one EN-BIRTH study hospital in Tanzania. BMC Pregnancy Childbirth 2021;21(1):1-11.

22. Channon AA, Padmadas SS, McDonald JW. Measuring birth weight in developing countries: does the method of reporting in retrospective surveys matter? Matern Child Health J 2011;15(1):12-18.

23. Blanc AK, Wardlaw T. Monitoring low birth weight: an evaluation of international estimates and an updated estimation procedure. Bulletin of the World Health Organization 2005;83(3):178-185.

24. Bollen KA, Noble MD, Adair LS. Are gestational age, birth weight, and birth length indicators of favorable fetal growth conditions? A structural equation analysis of Filipino infants. Stat Med 2013;32(17):2950-2961. 
25. Cutland CL, Lackritz EM, Mallett-Moore T, Bardají A, Chandrasekaran R, Lahariya C, et al. Low birth weight: Case definition \& guidelines for data collection, analysis, and presentation of maternal immunization safety data. Vaccine 2017 Dec 04,;35(48):6492-6500.

26. Abdel-Rahman SM, Paul IM, Delmore P, Chen J, Mills M, Greenberg RG. Validation and human factor analysis study of an infant weight estimation device. BMC Pediatr 2020;20(1):1-8.

27. Kong S, Day L, Bin Zaman S, Peven K, Salim N, Sunny AK, et al. Birthweight: EN-BIRTH multi-country study. BMC Pregnancy Childbirth 2020.

28. Exploring the information and ICT skills of health professionals in low-and middle-income countries. International Conference on Stakeholders and Information Technology in Education: Springer; 2016.

29. WHO, UNICEF. Every Newborn: an action plan to end preventable deaths. 2014.

30. Assembly G. Sustainable development goals. SDGs Transform Our World 2015;2030.

31. Chiba Y, Oguttu MA, Nakayama T. Quantitative and qualitative verification of data quality in the childbirth registers of two rural district hospitals in Western Kenya. Midwifery 2012;28(3):329-339.

32. Kenya National Bureau of Statistics, Ministry of Health/Kenya, National AIDS Control Council/Kenya, Kenya Medical Research Institute, National Council for Population and Development/Kenya. Kenya Demographic and Health Survey 2014. 2015.

33. Mvunta MH, Mboya IB, Msuya SE, John B, Obure J, Mahande MJ. Incidence and recurrence risk of low birth weight in Northern Tanzania: A registry based study. Plos One 2019;14(4).

34. Kamala BA, Mgaya AH, Ngarina MM, Kidanto HL. Predictors of low birth weight and 24-hour perinatal outcomes at Muhimbili National Hospital in Dar es Salaam, Tanzania: a five-year retrospective analysis of obstetric records. Pan Afr Med J 2018;29(1):1-13.

35. East LA, Arudo J, Loefler M, Evans CM. Exploring the potential for advanced nursing practice role development in Kenya: a qualitative study. BMC Nursing 2014;13(1):1-11.

36. Marete I, Ekhaguere O, Bann CM, Bucher SL, Nyongesa P, Patel AB, et al. Regional trends in birth weight in low-and middle-income countries 2013-2018. Reprod Health 2020;17(3):1-8.

37. Rekha C, Whelan RM, Reddy P, Reddy PS. Evaluation of adjustment methods used to determine prevalence of low birth-weight babies at a rural hospital in Andhra Pradesh, India. Indian J Public Health 2013;57(3):177.

38. Ministry of Health, Community Development, Gender, Elderly and Children - MoHCDGEC/Tanzania Mainland, Ministry of Health - MoH/Zanzibar, National Bureau of Statistics - NBS/Tanzania, Office of Chief Government Statistician - OCGS/Zanzibar, ICF. Tanzania Demographic and Health Survey and Malaria Indicator Survey 2015-2016. 2016.

39. Mulissa Z, Wendrad N, Bitewulign B, Biadgo A, Abate M, Alemu H, et al. Effect of data quality improvement intervention on health management information system data accuracy: An interrupted time series analysis. Plos One 2020;15(8).

40. Bhattacharya AA, Allen E, Umar N, Audu A, Felix H, Schellenberg J, et al. Improving the quality of routine maternal and newborn data captured in primary health facilities in Gombe State, 
Northeastern Nigeria: a before-and-after study. BMJ Open 2020;10(12).

41. Mphatswe W, Mate KS, Bennett B, Ngidi H, Reddy J, Barker PM, et al. Improving public health information: a data quality intervention in KwaZulu-Natal, South Africa. Bull World Health Organ 2012;90:176-182.

42. Day LT, Gore-Langton G, Rahman AE, Basnet O, Shabani J, Tahsina T, et al. Labour and delivery ward register data availability, quality, and utility - Every Newborn - birth indicators research tracking in hospitals (EN-BIRTH) study baseline analysis in three countries. BMC Health Serv Res 2020;20(1):737.

43. Baye E, Abate FW, Eglovitch M. Shiferie F, Olson IE, Shifraw T, et al. Effect of birthweight measurement quality improvement on low birthweight prevalence in rural Ethiopia. Popul Health Metrics 2021;19:35.

\section{Figures}

\section{Figure 1}

Study design: a prospective intervention study with historical controls

\section{Figure 2}

Birth weight distribution in Kenya and Tanzania: Prospective and historical sample 